\title{
Kinetics and Monte Carlo simulation of UV disinfection of $B$. subtilis spores and SARS-CoV-2 in dried saliva droplets
}

\author{
John Gibson ${ }^{1}$ (D) Ramin Farnood ${ }^{2} \cdot$ Benoit Barbeau $^{3}$ \\ Received: 6 July 2021 / Accepted: 10 September 2021 / Published online: 1 October 2021 \\ (C) The Author(s), under exclusive licence to Springer-Verlag GmbH Germany, part of Springer Nature 2021
}

\begin{abstract}
Surfaces can be contaminated by droplets produced through coughing or sneezing. In this exploratory work, the UV disinfection results of Bacillus subtilis spores in dried saliva droplets were fitted to a three-parameter kinetic model $\left(R^{2} \geq 0.97\right)$. This model has a disinfection rate constant for single organisms and a smaller one for aggregates found in droplets. The fraction of organisms found in aggregates $(\beta)$ could account for the effects of different-sized droplets in the experimental work. Since a wide spectrum of droplet sizes can be produced, and some of the rate constants were uncertain, Monte Carlo simulation was used to estimate the UV inactivation performance in dried saliva droplets in a variety of conditions. Using conservative distribution for $\beta$, the model was applied to the UV disinfection of SARS-CoV-2 in dried saliva droplets. It was shown that a one-log reduction of SARSCoV-2 was very likely ( $p>99.9 \%)$ and a two-log reduction was probable $(p=75 \%)$ at a dose of $60 \mathrm{~mJ} / \mathrm{cm}^{2}$. Aggregates tend to be variable and limit the log reductions that can be achieved at high UV doses.
\end{abstract}

Keywords SARS-CoV-2 · COVID-19 · UV disinfection · Kinetics · Surface disinfection

\section{Introduction}

We are in what is now, hopefully, the final stages of a global COVID-19 pandemic, caused by the severe acute respiratory syndrome coronavirus 2 (SARS-CoV-2). This is the latest in a series of coronavirus outbreaks that also include severe acute respiratory syndrome (SARS-CoV-1) and Middle East respiratory syndrome (MERS)(Noorimotlagh 2020). It is reasonable to expect more outbreaks in the future, and disinfection of these viruses is an important research area.

Responsible Editor: Lotfi Aleya

John Gibson

jh.gibson@utoronto.ca

1 Department of Chemical Engineering and Applied Chemistry, University of Toronto, 200 College St., Toronto, ON M5S 3E5, Canada

2 Department of Chemical Engineering and Applied Chemistry, University of Toronto, 200 College St, Toronto, ON M5S 3E5, Canada

3 Department of Civil, Geological, and Mining Engineering, Polytechnique Montréal, 2500 Chemin de Polytechnique, Montréal, Québec H3T 1J4, Canada
UV radiation, particularly at a wavelength of $254 \mathrm{~nm}$, has long been understood to be absorbed by the pyrimidine bases, thymine in DNA, and uracil in RNA, causing dimers that slow or halt reproduction (Jagger 1967; Masschelein 2002). When it comes to viruses, Pfaender et al. (2015) showed direct damage of the hepatitis $\mathrm{C}$ virus genome, slowing replication after UV treatment. Beck et al. (2016) showed RNA damage of the bacteriophage MS2 after UV treatment at wavelengths of 210 and $290 \mathrm{~nm}$.

Many viruses, including SARS-CoV-2, have been shown to persist on surfaces for several days (Noorimotlagh 2020). Sources of surface contamination included droplets produced by speaking, sneezing, or coughing (Dbouk and Drikakis 2020; Johnson et al. 2011). Faezeh seif et al. (2021) showed that not only were 40 of 76 hospital surface samples (e.g., bed rails and door handles) positive for SAR-CoV-2, they suggest possible selection for strains resistant to traditional disinfectants, such as ethanol and sodium hypochlorite.

The ability of UV to disinfect viral pathogens in dried droplets remains largely absent from the current literature. A recent metaanalysis by Chiappa et al. (2021) reports that just $11 \%$ (2 of 18) of the selected studies examined the effects of UV on viruses in dried droplets, with the majority (14 of 18) using liquid suspensions. The two dried droplets studies focused on demonstrating new technologies: a rotating full-room disinfector (Bedell et al. 
2016) and a pulsed xenon emitter (Simmons et al. 2021). Though both studies were able to show reductions in coronaviruses, their unique emitters make it difficult to apply these results elsewhere. Ma et al. (2021), after exploring the UV disinfection in liquid suspension of the MHV, $\Phi 6$, and coronavirus HCoV 299E, suggested a need for better understanding UV disinfection on surfaces. Overall, UV disinfection of contaminated surfaces remains poorly understood.

Experimental work on dried droplets is challenging due to the small size of the droplets. Johnson et al. (2011) showed that the largest droplets produced by speaking had a mode diameter of just $145 \mu \mathrm{m}$, corresponding to a volume of just $0.0016 \mu \mathrm{L}$. Lab work with such small volumes is challenging. The ASTM test (2018) E3135 - 18 Standard Practice for Determining Antimicrobial Efficacy of Ultraviolet Germicidal Irradiation Against Microorganisms on Carriers with Simulated Soil suggests using droplets of $1 \mu \mathrm{L}$ instead. ASTM test (2016) E2721 - 16 Standard Practice for Evaluation of Effectiveness of Decontamination Procedures for Surfaces When Challenged with Droplets Containing Human Pathogenic Viruses aims to produce droplets $15 \mu \mathrm{m}$ in diameter. However, this is done by atomizing the virus, which is a safety concern if viruses escape before, during, or after the experiment. Significant losses are observed during the aerosolization procedure. In addition, Barancheshme et al. (2021) showed there is wide variability in the UV transmission of human saliva, another source of variability in experiments. These factors make experimental work challenging.

Computer modeling can inform us about UV disinfection of droplets expelled by infected individuals on surfaces without some of the challenges associated with experimental work. Monte Carlo simulation (MCS) is a modelling approach that allows the variables in a model to take on statistical distributions to account for uncertainty or variability. The model is executed many thousands of times, each time taking a random sample of the variables, and the ability to meet some performance objective is tabulated. In this case, MCS can be used to account for the wide distribution of droplet characteristics and uncertainty in the disinfection rate constants. The performance measure in this work is the number of log reductions that can be achieved at a given UV dose.

Experimental studies on dried droplets are rare, and we are aware of no previous work using MSC to characterize their UV disinfection behavior. As such, the objective of this work is to first develop a kinetic model of UV disinfection in dried droplets and then use this model to better characterize disinfection of viruses on surfaces using Monte Carlo simulation.

\section{Methods and model development}

Disinfection is typically modelled as a first-order process where the rate of change of the number of surviving organisms is proportional to the dose received. The ratio of surviving organism $\left(N / N_{\mathrm{o}}\right)$ is given by:

$\frac{N}{N_{o}}=e^{-k D}$

The dose $(D)$ is the product of the light intensity $\left(\mathrm{mW} / \mathrm{cm}^{2}\right)$ the organism receives and the duration of exposure (s). The parameter $k$ is a disinfection rate constant $\left(\mathrm{cm}^{2} / \mathrm{mJ}\right)$.

Tseng and Li (2007) showed good agreement with the firstorder model when disinfecting viruses with UV on surfaces, with $R^{2}$ values of $0.94,0.96,0.96$, and 0.98 for MS2, $\Phi X 174$, $\Phi 6$, and T7 viruses, respectively. The lower UV sensitivity of viruses on surfaces when compared to aerosols observed by Tseng and $\mathrm{Li}$ (2007) was postulated to involve the presence of aggregates on the surface, which is addressed below.

Aggregates of organisms, often associated with small particles, are a historical problem in UV disinfection (Azimi et al. 2012, 2013; Emerick et al. 2000; Loge 1996). Mathematical models developed to describe this behavior include that of Emerick et al. (2000), which introduced a parameter for the number of particle-associated organisms $\left(N_{p}\right)$. Since they are harder to disinfect, the rate of inactivation of $N_{p}$ was assumed to be linear with time, rather than exponential.

Other authors have used double-exponential models where the process remains first order, with different exponential rate constants for single organisms and a smaller one for particleassociated aggregates (Azimi et al. 2012; Barbeau et al. 2005). The model used in this work, of the double-exponential type, is adapted from Barbeau et al. (2005) and shown below:

$\frac{N}{N_{o}}=(1-\beta) e^{-k_{S} D}+\beta e^{-k_{A} D}$

In this model, $k_{S}$ is the rate constant for single organisms; $k_{A}$ is the rate constant for aggregates; $D$ is the UV dose; and $\beta$ is the fraction of the population found in aggregates. The rate constant for aggregates is smaller than that of individual organisms, consistent with the fact that they are harder to disinfect. There is most likely a distribution of rate constants (i.e., more than two), but the current scientific literature precludes us from defining rate constants based on aggregate characteristics. One advantage of this relatively simple model is the small number of parameters that must be estimated. This model can also help identify the aggregate characteristics that control the value of $k_{A}$. 


\section{Monte Carlo simulation}

Monte Carlo simulation can be used to account for variability and uncertainty. In this work, the model is executed 10,000 times, each time taking a random sample of the uncertain variables. Increasing the number of model executions to 15,000 produced results that agreed with 10,000 executions within $1 \%$, suggesting a sufficient number had been used. The results are tabulated to see what fraction of a given log reduction in $N / N_{\mathrm{o}}$ is exceeded at some UV dose. This allows an estimate of UV performance as a disinfectant, even if some of the parameters are uncertain or variable.

The most uncertain parameter in this work is the fraction of the viral population in aggregates ( $\beta$ ). Johnson et al. (2011) showed that the diameter of saliva droplets produced by speaking followed a lognormal distribution, so $\beta$ is also assumed to be lognormal. Since there is a high degree of uncertainty in estimating $\beta$, a relatively large standard deviation is assumed for this lognormal distribution: $80 \%$ of the mean. Figure 1 shows the distribution of $\beta$ used in this work. Note that the mean value is about $2 \%$, but values as low as $0.3 \%$ and exceeding $7.0 \%$ are possible.

There is less uncertainty in the single organism disinfection rate constant $\left(k_{S}\right)$ since published values are often available. However, the aggregate rate constant $\left(k_{A}\right)$, as discussed previously, is more uncertain, with few values found in the literature. To account for this uncertainty, $k_{A}$ is assumed to be normally distributed with a standard deviation of $50 \%$ of the mean.

\section{SARS-CoV-2 modelling}

One of the advantages of the relatively simple model used here is that only three parameters must be estimated to examine the UV response of other pathogens: $k_{S}, k_{A}$, and $\beta$. Based on the recent review of Hessling et al. (2020), the median observed value for $k_{S}$ for several different coronaviruses is approximately $0.22 \mathrm{~cm}^{2} / \mathrm{mJ}$. This is consistent with the observed cluster of values for SARS-CoV-1, MS2, and $\Phi 6$ viruses published by Blatchley et al. (2020) $\left(18 \mathrm{~cm}^{2} / \mathrm{mJ}\right)$. Taking the average, a value of $0.20 \mathrm{~cm}^{2} / \mathrm{mJ}$ is used for the $k_{S}$ of SARS-CoV-2 in this work.

From the model validation work, described below, the values of $\beta$ were observed to be $2 \%$ and $13 \%$ for $2-\mu \mathrm{L}$ and $10-\mu \mathrm{L}$ droplets, respectively. This suggests $\beta$ decreases as droplets get smaller. The actual droplets we are interested in are much smaller than $2 \mu \mathrm{L}$, on the order of $0.002 \mu \mathrm{L}$. Nonetheless, we will conservatively assume a mean value for $\beta$ of 2\% for disinfection of SARS-CoV-2. This ensures there is some safety factor in any disinfection predictions.

Estimating $k_{A}$ for SARS-CoV-2 is difficult, but there is some previous work on particle-associated bacteria on which we can draw. Azimi et al. (2012) observed a ratio of $k_{A} / k_{S}$ of 6\%. Kollu and Örmeci (2012) observed an approximate ratio of $k_{A} / k_{S}$ of $15 \%$. The ratio of $k_{A} / k_{S}$ in the model validation in this work was $12 \%$. Therefore, we assign a median value of $k_{A} / k_{S}$ of $10 \%$ in this work based on other observations. This gives a value of $k_{A}$ of $0.020 \mathrm{~cm}^{2} / \mathrm{mJ}$ for SARS-CoV-2 (10\% of $\left.k_{S}\right)$. Formulating $k_{A}$ in this way ensures that it is proportional to the single organism rate constant.

Table 1 shows an overview of the model inputs used for Bacillus subtilis spores and SARS-CoV-2.

\section{Results}

\section{Model validation}

Less hazardous surrogates are often substituted for more dangerous ones in laboratory work. The work of Barancheshme et al. (2021) used relatively difficult to disinfect, but harmless, B. subtilis spores in dried human saliva droplets. Figure 2 shows the results of Barancheshme et al. (2021) on
Fig. 1 Distribution of the fraction of the population found in aggregates $(\beta)$ used in this work $(10,000$ simulations $)$

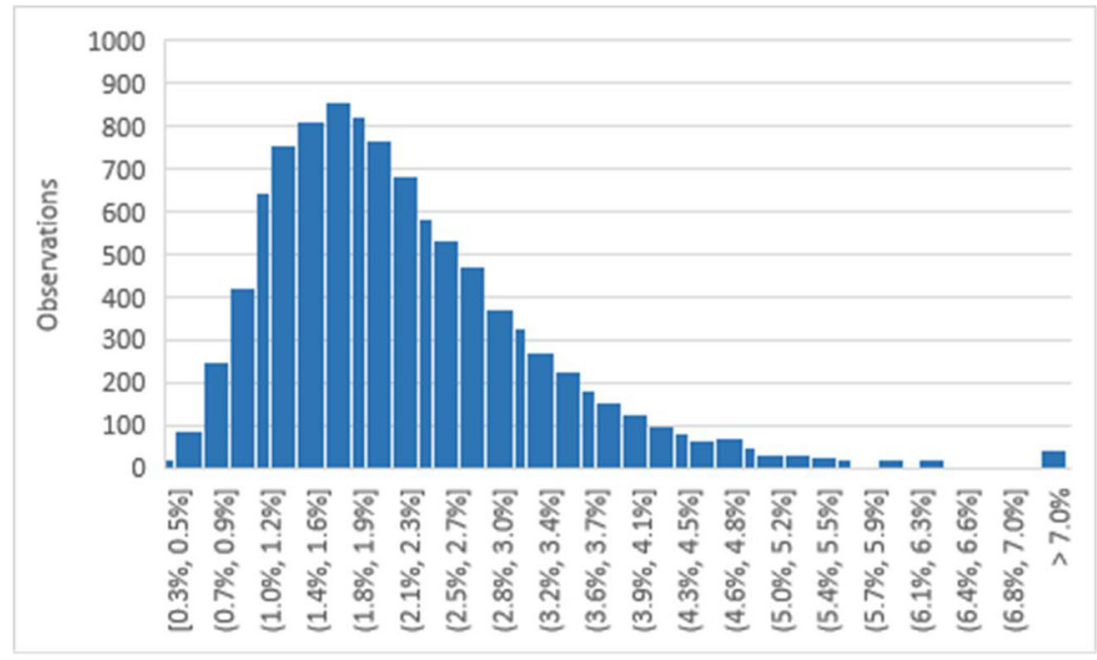


Table 1 Monte Carlo simulation parameters

\begin{tabular}{lllll}
\hline Test organism & Model parameter & Distribution type & Mean value & Standard deviation (\% of mean) \\
\hline B. subtilis spores & Single organism rate constant $\left(k_{s}\right)$ & $\mathrm{n} / \mathrm{a}$ & $0.10 \mathrm{~cm}^{2} / \mathrm{mJ}$ & $\mathrm{n} / \mathrm{a}$ \\
& Aggregate disinfection rate constant $\left(k_{A}\right)$ & Normal & $0.012 \mathrm{~cm}^{2} / \mathrm{mJ}$ & $0.006(50 \%)$ \\
& Fraction of particle-associated organisms $(\beta)$ & Lognormal & $2 \%$ & $1.8 \%(80 \%)$ \\
SARS-CoV-2 & Single organism rate constant $(k s)$ & $\mathrm{n} / \mathrm{a}$ & $0.20 \mathrm{~cm}^{2} / \mathrm{mJ}$ & $\mathrm{n} / \mathrm{a}$ \\
& Aggregate disinfection rate constant $\left(k_{A}\right)$ & Normal & $0.020 \mathrm{~cm}^{2} / \mathrm{mJ}$ & $0.010(50 \%)$ \\
& Fraction of particle-associated organisms $(\beta)$ & Lognormal & $2 \%$ & $1.8 \%(80 \%)$
\end{tabular}

See "Methods and model development" section for details of parameter estimation

B. subtilis spores fitted to a double-exponential model for $2-\mu \mathrm{L}$ and $10-\mu \mathrm{L}$ droplets. Both rate constants $\left(k_{S}\right.$ and $\left.k_{A}\right)$ have the same values independent of droplet size. The only difference between the figures is the fraction of organisms found in aggregates $(\beta)$, which decreases with droplet size from 13 to $2 \%$. The two-population, double-exponential model effectively described the UV disinfection of $B$. subtilis spores in dried human saliva resulting in $R^{2}$ values of 0.98 and 0.97 for $2-\mu \mathrm{L}$ and $10-\mu \mathrm{L}$ droplets, respectively. These results suggest that the rate constants are indeed constant, and the effects of droplet size can be approximated by changing the $\beta$ alone.

\section{Monte Carlo simulation of $B$. subtilis spore UV inactivation}

The number of organisms associated with aggregates is both variable and uncertain, since no two coughs are the same. Based on the model validation, this variability is approximated by changing the parameter $\beta$. The precise value of $k_{A}$ is also not known, only approximated, so a normal distribution is used to account for this. Allowing for this uncertainty, Table 2 shows the expected log reduction in $B$. subtilis spores in dried saliva droplets.
Fig. 2 UV disinfection of B. subtilis spores in human saliva in dried $2-\mu \mathrm{L}(\mathbf{A})$ and $10-\mu \mathrm{L}(\mathbf{B})$ droplets fitted to a doubleexponential model $\left(k_{S}=\right.$ rate constant for single organisms; $k_{A}=$ rate constant for aggregates; $\beta=$ fraction of organisms in aggregates)
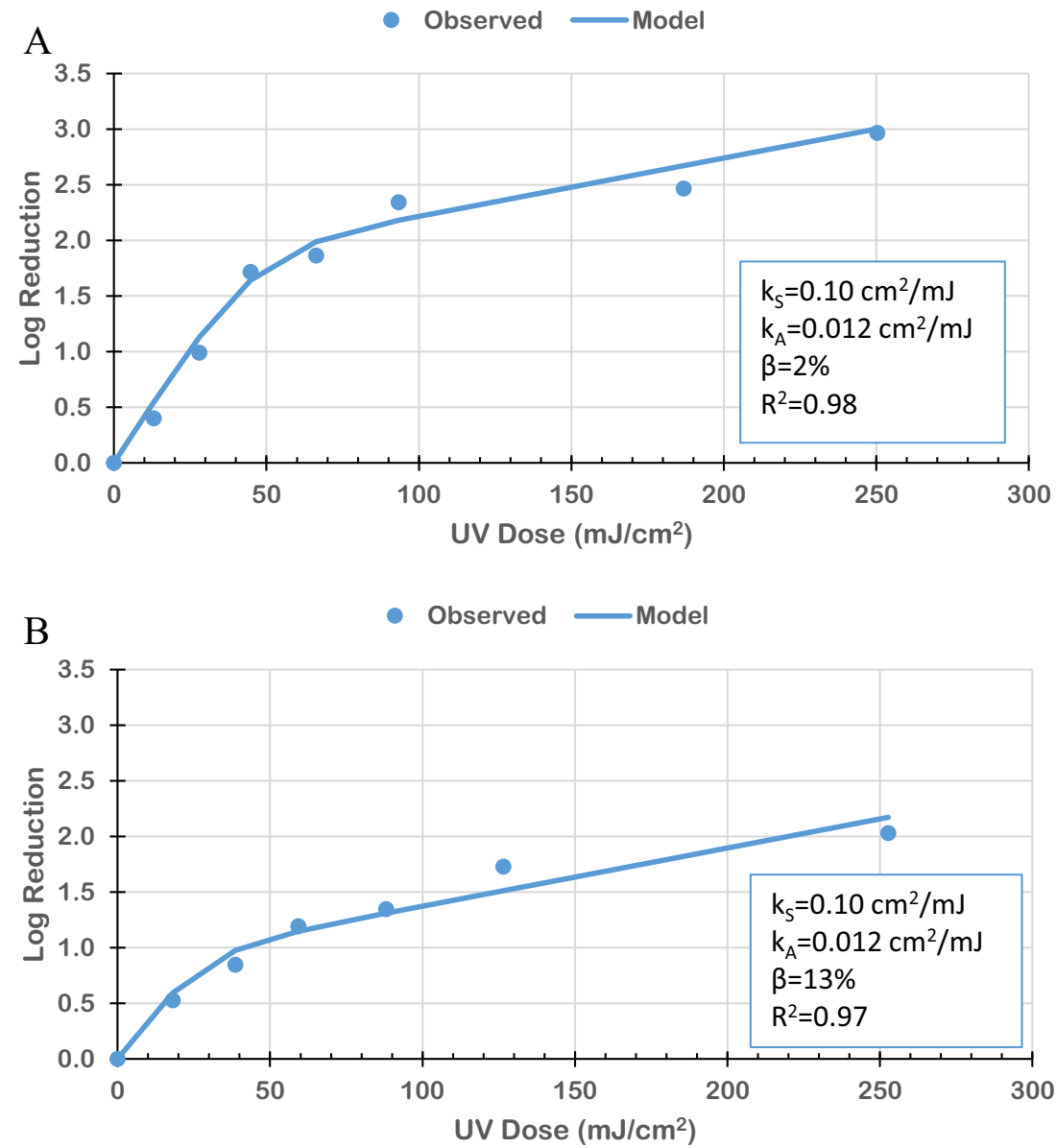
The results of the MCS show that there are diminishing returns as UV dose is increased. One-log reduction can be achieved at a dose of $40 \mathrm{~mJ} / \mathrm{cm}^{2}$. However, doubling this dose to $80 \mathrm{~mJ} / \mathrm{cm}^{2}$ shows that a two-log reduction will be achieved only $64 \%$ of the time. Tripling the dose to $120 \mathrm{~mJ} / \mathrm{cm}^{2}$ only increases the chances of two-log (or more) reduction to just $81 \%$. The log reductions that can be achieved get more difficult at higher UV doses.

One reason for this is that the rate constant for aggregates, important at higher doses, is an order of magnitude smaller than that of single organisms. Also, aggregates are variable in this model, and it gets more unlikely to achieve complete inactivation at the higher doses where they dominate. For example, some of the time, aggregates will contain such a large fraction of the total population (e.g., $>5 \%$ ) that a high $\log$ reduction is difficult to achieve.

\section{Monte Carlo simulation of SARS-CoV-2 inactivation}

The single organism rate constant $\left(k_{S}\right)$ for SARS-CoV-2 used in this work is twice that of B. subtilis spores $\left(0.20\right.$ vs. $\left.0.10 \mathrm{~cm}^{2} / \mathrm{mJ}\right)$. In addition, the aggregate rate constant $\left(k_{A}\right)$ is assumed to be proportional to the single organism disinfection sensitivity $\left(k_{S}\right)$, making both rate constants larger. These higher rate constants make the $\log$ reductions that can be achieved with SARS-CoV-2 greater than that of B. subtilis spores (Table 3).

For example, a one-log reduction in SARS-CoV-2 is very likely $\left(p>99.9 \%\right.$ ) at a dose of just $20 \mathrm{~mJ} / \mathrm{cm}^{2}$, but very unlikely for $B$. subtilis spores $(p<0.1 \%)$. At $40 \mathrm{~mJ} /$ $\mathrm{cm}^{2}$, the probability of exceeding a two-log reduction in SARS-CoV-2 is $55 \%$, where it is $<0.1 \%$ for $B$. subtilis spores. Overall, this makes $B$. subtilis spores a conservative surrogate for SARS-CoV-2.

Once again, even for the more UV-sensitive SARSCoV-2, aggregates tend to limit the ability to achieve high $\log$ reductions. Doubling the dose from 60 to $120 \mathrm{~mJ} / \mathrm{cm}^{2}$ increases the probability of two-log reduction from 75 to $91 \%$, an increase of just $17 \%$. The probability of achieving greater than a three-log reduction never exceeds $33 \%$. This suggests the three-log reductions are likely difficult to achieve in this conservative model. In practical terms, a

Table 2 Probability of achieving different log reductions in B. subtilis spores as a function of UV dose (Monte Carlo simulation with 10,000 model executions)

\begin{tabular}{lllllll}
\hline & \multicolumn{5}{l}{ Dose $\left(\mathrm{mJ} / \mathrm{cm}^{2}\right)$} \\
\cline { 2 - 7 } Log reduction & 20 & 40 & 60 & 80 & 100 & 120 \\
\hline$>1 \log$ & $<0.1 \%$ & $>99.9 \%$ & $>99.9 \%$ & $>99.9 \%$ & $>99.9 \%$ & $>99.9 \%$ \\
$>2 \log$ & $<0.1 \%$ & $<0.1 \%$ & $33 \%$ & $64 \%$ & $75 \%$ & $81 \%$ \\
$>3 \log$ & $<0.1 \%$ & $<0.1 \%$ & $<0.1 \%$ & $<0.1 \%$ & $1 \%$ & $4 \%$ \\
\hline
\end{tabular}

Table 3 Probability of achieving different log reductions in SARSCoV-2 as a function of UV dose (Monte Carlo simulation with 10,000 model executions)

\begin{tabular}{lllllll}
\hline & \multicolumn{5}{l}{ Dose $\left(\mathrm{mJ} / \mathrm{cm}^{2}\right)$} \\
\cline { 2 - 7 } $\begin{array}{c}\log \\
\text { reduction }\end{array}$ & 20 & 40 & 60 & 80 & 100 & 120 \\
\hline$>1 \log$ & $>99.9 \%$ & $>99.9 \%$ & $>99.9 \%$ & $>99.9 \%$ & $>99.9 \%$ & $>99.9 \%$ \\
$>2 \log$ & $<0.1 \%$ & $55 \%$ & $75 \%$ & $84 \%$ & $89 \%$ & $91 \%$ \\
$>3 \log$ & $<0.1 \%$ & $<0.1 \%$ & $1 \%$ & $7 \%$ & $19 \%$ & $33 \%$ \\
\hline
\end{tabular}

two-log reduction of SARS-CoV-2 in dried saliva droplets is likely a reasonable target.

According to the model, a one-log reduction is very likely $(>99.9 \%)$ and a two-log reduction is probable $(p=75 \%)$ for SARS-CoV-2 at a dose of $60 \mathrm{~mJ} / \mathrm{cm}^{2}$. This is likely a practical dose to aim for. Figure 3 shows the distribution of log reductions at $60 \mathrm{~mJ} / \mathrm{cm}^{2}$. Note that both low $(<1.4)$ and high $(>3.2)$ $\log$ reductions are observed, illustrating the variable nature of dried droplets in this model.

\section{Model limitations}

The assumptions of the model are described in the "Methods and model development" section. Briefly, we assume that UV disinfection of dried saliva is like UV disinfection in other liquids containing aggregates and/or particles. The "Model validation" section shows that the observed disinfection of B. subtilis spores in dried saliva could be described with $R^{2}$ $\geq 0.97$. The fraction of organisms in aggregates is estimated using a single parameter $\beta$, but the situation is likely much more complex. However, even if $\beta$ could be determined experimentally, there is no guarantee that a different cough or sneeze would produce the same result. Nonetheless, a better understanding how viruses are distributed in dried droplets could improve this model. The single organism rate constant for SARS-CoV-2, on which so much depends, is approximate and based on the literature and not actual experiments. This also could be improved.

Lastly, the validation work was done using bacterial spores, and this information was extrapolated to viruses. This size discrepancy between viruses and bacteria likely contributes more uncertainty in $\beta$ and $k_{A}$ for SARSCoV-2. However, some uncertainty in these parameters was accounted for in the MCS. In addition, an appropriate single organism rate constant was used for SARSCoV-2, and $k_{S}$ describes most of the behavior at low $\log$ reductions. High $\log$ reductions are uncertain, and this is reflected in the model. Overall, a double-exponential, three-parameter model that can describe UV disinfection in dried saliva droplets is proposed. 
Fig. 3 Log reductions of SARSCoV-2 in dried saliva droplets at a UV dose of $60 \mathrm{~mJ} / \mathrm{cm}^{2}(10,000$ model executions)

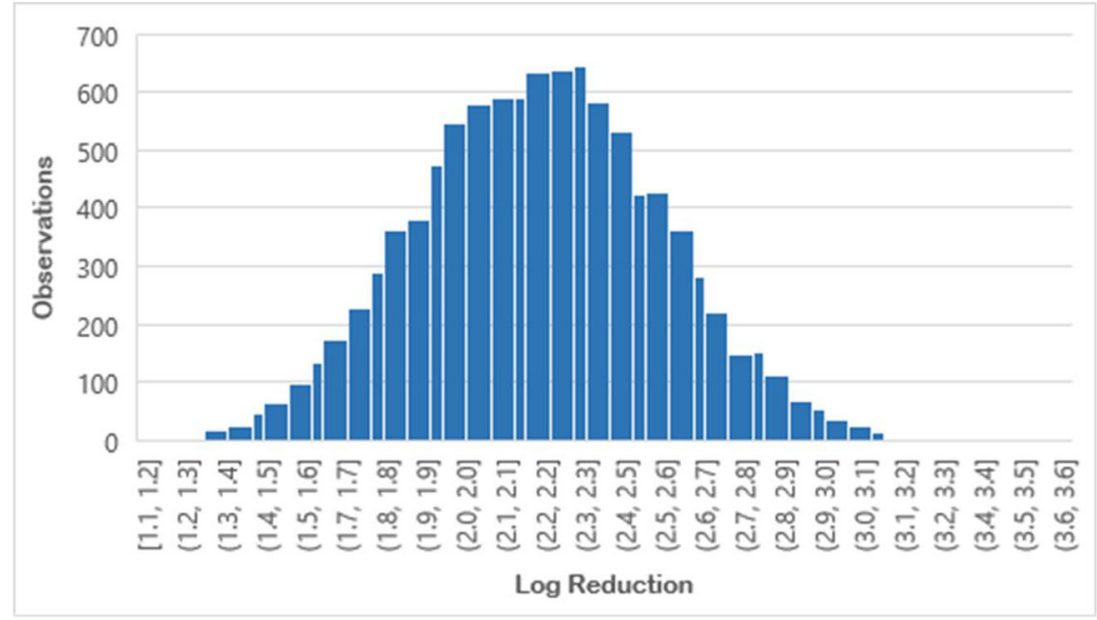

\section{Conclusions}

In this work, we demonstrated the UV disinfection of B. subtilis spores in dried human saliva could be described by a two-population, double-exponential model $\left(R^{2} \geq 0.97\right)$ where $\beta$ is the fraction of organisms in aggregates. The value of $\beta$ was $2 \%$ for dried $2-\mu \mathrm{L}$ droplets and decreased with droplet size. We assume that $\beta$ is variable and it was assigned a lognormal distribution. Monte Carlo simulation (MCS) of B. subtilis spores and SARS-CoV-2 showed increases in the resistance to UV disinfection and increases in the variability in the results at higher $\log$ reductions. This was attributed to the lower disinfection rate constant for aggregates $\left(k_{A}\right)$ and the variable nature of droplets. It was shown, using a conservative estimation of $\beta$ for very small droplets, that a two-log reduction of SARS-CoV-2 was probable $(p=75 \%)$ at a dose of 60 $\mathrm{mJ} / \mathrm{cm}^{2}$. Higher log reductions are more uncertain and difficult to achieve. Future work should investigate the impact of dried droplets characteristics on the kinetic parameters of the double-exponential model.

Author contribution JG conceptualized work, did the numerical analysis, and wrote the first draft of report.

$\mathrm{RF}$ provided editorial comments on the draft repot.

BB provided experimental data used in validating model and editorial comments and suggestions for draft report.

Funding Funding was provided by Michael and Teresa Wu COVID-19 Research Fund Award and University of Toronto Connaught Fund.

Data availability The datasets used and/or analyzed during the current study are available from the corresponding author on reasonable request.

\section{Declarations}

Ethics approval and consent to participate This study did not involve human participants, human data, or human tissue.
Consent for publication Consent was provided by one the co-authors of this study (Barbeau) to use experimental results from his earlier study (Barancheshme et al. 2021).

Competing interests The authors declare no competing interests.

\section{References}

American Society for Testing Materials (ASTM) (2016) E2721 - 16 Standard practice for evaluation of effectiveness of decontamination procedures for surfaces when challenged with droplets containing human pathogenic viruses.

American Society for Testing Materials (ASTM) (2018) E3135 - 18 Standard practice for determining antimicrobial efficacy of ultraviolet germicidal irradiation against microorganisms on carriers with simulated soil

Azimi Y, Allen DG, Farnood RR (2012) Kinetics of UV inactivation of wastewater bioflocs. Water Res 46:3827-3836. https://doi.org/10. 1016/j.watres.2012.04.019

Azimi Y, Chen X, Allen DG, Pileggi V, Seto P, Droppo IG, Farnood RR (2013) UV disinfection of wastewater flocs: the effect of secondary treatment conditions. Water Sci Technol 67:2719-2723. https://doi. org/10.2166/wst.2013.148

Barancheshme F, Philibert J, Noam-Amar N, Gerchman Y, Barbeau B (2021) Assessment of saliva interference with UV-based disinfection technologies. J Photochem Photobiol B 217:112168. https://doi. org/10.1016/j.jphotobiol.2021.112168

Barbeau B, Huffman D, Mysore C, Desjardins R, Clément B, Prévost M (2005) Examination of discrete and counfounding effects of water quality parameters during the inactivation of MS2 phages and Bacillus subtilis spores with chlorine dioxide. J Environ Eng Sci 4: 139-151. https://doi.org/10.1139/s04-050

Beck SE, Rodriguez RA, Hawkins MA, Hargy TM, Larason TC, Linden KG (2016) Comparison of UV-induced inactivation and RNA damage in MS2 phage across the germicidal UV spectrum. Appl Environ Microbiol 82:1468-1474. https://doi.org/10.1128/AEM. 02773-15

Bedell K, Buchaklian AH, Perlman S (2016) Efficacy of an automated multiple emitter whole-room ultraviolet-C disinfection system against coronaviruses MHV and MERS-CoV. Infect Control Hosp Epidemiol 37:598-599. https://doi.org/10.1017/ice.2015.348

Blatchley ER, Petri B, Sun W (2020)SARS-CoV-2 UV dose-response behavior. White Paper. International Ultraviolet Association (IUVA). Chevy Chase, MD 
Chiappa F, Frascella B, Vigezzi GP, Moro M, Diamanti L, Gentile L, Lago P, Clementi N, Signorelli C, Mancini N, Odone A (2021) The efficacy of UV light-emitting technology against coronaviruses: a systematic review. J Hosp Infect S0195670121002085:63-78. https://doi.org/10.1016/j.jhin.2021.05.005

Dbouk T, Drikakis D (2020) On coughing and airborne droplet transmission to humans. Phys Fluids 32:053310. https://doi.org/10.1063/5. 0011960

Emerick RW, Loge FJ, Ginn T, Darby JL (2000) Modeling the inactivation of particle-associated coliform bacteria. Water Environ Res 72: 432-438

Hessling M, Hones K, Vatter P, Lingenfelder C (2020) Ultraviolet irradiation doses for coronavirus inactivation - review and analysis of coronavirus photoinactivation studies. GMS Hyg Infect Control 15. https://doi.org/10.3205/dgkh000343

Jagger J (1967) Introduction to research in ultraviolet photobiology. Prentice-Hall, Englewood Cliffs

Johnson GR, Morawska L, Ristovski ZD, Hargreaves M, Mengersen K, Chao CYH, Wan MP, Li Y, Xie X, Katoshevski D, Corbett S (2011) Modality of human expired aerosol size distributions. J Aerosol Sci 42:839-851. https://doi.org/10.1016/j.jaerosci.2011.07.009

Kollu K, Örmeci B (2012) Effect of particles and bioflocculation on ultraviolet disinfection of Escherichia coli. Water Res 46:750-760. https://doi.org/10.1016/j.watres.2011.11.046

Loge FJ (1996) Ultraviolet disinfection of secondary wastewater effluents: prediction of performance and design. Wat Environ Rsch 68: 900-916. https://www.jstor.org/stable/25044788

Ma B, Linden YS, Gundy PM, Gerba CP, Sobsey MD, Linden KG (2021) Inactivation of Coronaviruses and phage Phi6 from irradiation across UVC wavelengths. Environ Sci Technol Lett 8:425-430. https://doi.org/10.1021/acs.estlett.1c00178
Masschelein WJ (2002) Ultraviolet light in water and wastewater sanitation. Lewis Publishers, CRC Press, New York

Noorimotlagh Z (2020) A systematic review of emerging human coronavirus (SARS-CoV-2) outbreak: focus on disinfection methods, environmental survival, and control and prevention strategies. Environ Sci Pollut Res 15:1-15

Pfaender S, Brinkmann J, Todt D, Riebesehl N, Steinmann J, Steinmann J, Pietschmann T, Steinmann E (2015) Mechanisms of methods for hepatitis C virus inactivation. Appl Environ Microbiol 81:16161621. https://doi.org/10.1128/AEM.03580-14

Seif F, Noorimotlagh Z, Mirzaee SA, Kalantar M, Barati B, Fard ME, Fard NK (2021) The SARS-CoV-2(COVID-19) pandemic in hospital: an insight into environmental surfaces contamination, disinfectants' efficiency, and estimation of plastic waste production. Environ Res 202:111809. https://doi.org/10.1016/j.envres.2021. 111809

Simmons SE, Carrion R, Alfson KJ, Staples HM, Jinadatha C, Jarvis WR, Sampathkumar P, Chemaly RF, Khawaja F, Povroznik M, Jackson S, Kaye KS, Rodriguez RM, Stibich MA (2021) Deactivation of SARS-CoV-2 with pulsed-xenon ultraviolet light: implications for environmental COVID-19 control. Infect Control Hosp Epidemiol 42:127-130. https://doi.org/10.1017/ice.2020.399

Tseng C-C, Li C-S(2007) Inactivation of viruses on surfaces by ultraviolet germicidal irradiation. J Occup Environ Hyg 4:400-405. https:// doi.org/10.1080/15459620701329012

Publisher's note Springer Nature remains neutral with regard to jurisdictional claims in published maps and institutional affiliations. 